\title{
Temporary Teeth Caries Intensity of Children Aged 4 to 6 Years from the Town Varna, Bulgaria
}

\author{
Dr Dobrinka Damyanova PhD student ${ }^{1}$, Dr Sirma Angelova PhD student ${ }^{1}$, \\ Dr Teodora Targova-Dimitrova $\mathrm{PhD}^{2}$, Assoc. Prof. Dr Katerina Ivanova \\ c.m.sc. ${ }^{3}$, Prof. Krasimira Prodanova ${ }^{4}$ \\ ${ }^{1}$ Assistant Professor,Medical University-Varna, Bulgaria, Faculty Of Dental Medicine, Department Of \\ Pediatric Dental Medicine \\ ${ }^{2}$ Assistant Professor,Medical University-Varna, Bulgaria, Faculty Of Dental Medicine, Department Of \\ Pariodotology And Dental Implantology \\ ${ }^{3}$ Associate Professor,Medical University-Varna, Bulgaria, Faculty Of Dental Medicine, Department Of \\ Pediatric Dental Medicine \\ ${ }^{4}$ Professor, Technical University Of Sofia, Bulgaria
}

\begin{abstract}
:
Introduction: The World Health Organization (WHO) 2003 report stated nearly 60\%-90\% of school children and most adults in industrialized nations had dental caries.

Purpose: To conduct an study of dental caries intensity of temporary teeth of children from the town Varna aged 4 to 6 years.

Materials and Methods: The study included 300 children aged 4 to 6 years old. The children were divided into three groups. The first group consisted of 100 children, aged 4 years old. Second group consisted of 100 children, aged 5 years old. The third group consisted of 100 children, aged 6 years old. To reflect data from studies and oral status are made clinical maps, maps tailored to the WHO. After processing results and highlights of the actual study was conducted by the data processing package for mathematical and statistical analysis StatSoft, Inc., STATISTICA Manual (Data analysis software system), Version 10.0, 2010.

Results: In temporary teeth dmft mean 3,57. We found the highest prevalence of cavitated caries in the first and second molars, and the lowest - in the lower second incisors and first incisors.

Conclusion: To the ratio $3 d /$ If we can conclude that children are not covered by timely treatment of early caries lesions.
\end{abstract}

Keywords: intensity of caries, caries lesion, temporary teeth

\section{Introduction}

The World Health Organization (WHO) 2003 report stated nearly 60\%-90\% of school children and most adults in industrialized nations had dental caries [1]. This high prevalence has declined dramatically, but caries remains a significant problem in some developing nations [2]. Objectives: To conduct an study of dental caries intensity of temporary teeth of children aged 4 to 6 years from the town Varna.

\section{Materials and Methods}

The study included 300 children aged 4 to 6 years old. The children were divided into three groups. The first group consisted of 100 children, aged 4 years old. Second group consisted of 100 children, aged 5 years old. The third group consisted of 100 children, aged 6 years old.

Children are reviewed with a history and detailed registration of clinical status. After detailed in outpatient registration cards numbers in the clinical setting with polished precision brush each tooth surfaces smooth, then dried. Investigates any smooth surface-vestibular and visual oral, clinical methods and register values obtained for each and every tooth surface The every child - patient.

1. Subject of research - caries of temporary teeth

2. The object of the study are: Children aged 4-6, the criteria for inclusion of children: From 4 to 6 year old children - healthy, accompanied and cared for by their parents, without systemic, stomatitis and gingival diseases. Regularly attending outpatient surgery with regular oral hygiene, dietary and prophylactic treatments.

Conduct and organization of study- survey period 2014-2015. The study is realized in the Faculty of Dental Medicine -Varna, Bulgaria. The study has been authorized by the Commission on the Ethics of Scientific Research at MU Varna and was taken in advance declared informed consent from each parent, or of any child patient. After processing results and highlights of the actual study was conducted by the data processing package 
for mathematical and statistical analysis StatSoft, Inc., STATISTICA Manual (Data analysis software system), Version 10.0, 2010 [3], in the period March-May 2016.

The method applied for the realization of the purpose and objectives of the study: Documentary method collecting data on the enrolled children eligible for inclusion.

To reflect data from studies and oral status are made clinical maps, maps tailored to the WHO. Epidemiological investigation and taking of oral status is carried out by $\mathrm{PhD}$ student with the help of a nurse assistant in a dental office, in clinical halls of the dental chair, directed light, water and air, individual sterile instruments - mirror without applying probe for lesions $\mathrm{d} 1 \mathrm{a}, \mathrm{d} 1 \mathrm{~b}, \mathrm{~d} 2$. Since acute probe is not used in these lesions probe ball WHO applies only to lesions with cavitation available. Diagnosis of caries lesions is done through visual observation, a view with an initial diagnostic threshold $\mathrm{d} 1 \mathrm{~d}(\mathrm{a}, \mathrm{d} 1 \mathrm{~b}, \mathrm{~d} 2)$ in vestibular and oral surfaces, reflecting the earliest identifiable visual lesions.

\section{Results}

Intensity of tooth decay of the studied children (Table 1, Table 2).

Table 1. The Intensity of caries by age

\begin{tabular}{|c|c|c|c|}
\hline \multicolumn{4}{|c|}{ Frequency Distribution of Age } \\
\hline Years & $\mathbf{N}$ & $\begin{array}{l}\text { Cumulative } \\
\text { frequency }\end{array}$ & \%From everyone \\
\hline 4 & 100 & 101 & 33,77926 \\
\hline 5 & 100 & 199 & 32,77592 \\
\hline 6 & 100 & 299 & 33,44482 \\
\hline
\end{tabular}

Table 2. Frequency distribution of the sex of the children from the three groups

\begin{tabular}{|l|l|l|l|}
\hline \multicolumn{4}{|l|}{ Frequency distribution of the sex of the children } \\
\hline Groups & N & Cumulative frequency & \% From everyone \\
\hline Boys & 160 & 159 & 53,17726 \\
\hline Girls & 140 & 299 & 46,82274 \\
\hline
\end{tabular}

Oral status of children studied: Table 3, Fig. 1.

Table 3. General distribution of caries of all surveyed children from groups

\begin{tabular}{|l|l|l|l|l|l|l|l|l|l|l|l|l|l|}
\hline $\begin{array}{l}\text { To } \\
\text { tal }\end{array}$ & $\mathbf{0}$ & $\mathbf{1}$ & $\mathbf{2}$ & $\mathbf{3}$ & $\mathbf{4}$ & $\mathbf{5}$ & $\mathbf{6}$ & $\mathbf{7}$ & $\mathbf{8}$ & $\mathbf{9}$ & $\mathbf{1 0}$ & $\mathbf{1 1}$ & $\mathbf{1 2}$ \\
\hline $\begin{array}{l}29 \\
9\end{array}$ & 93 & 71 & 25 & 16 & 28 & 16 & 24 & 8 & 6 & 3 & 7 & 1 & 1 \\
\hline & 31.1 & 23.7 & 8.36 & 5.3 & 9.3 & 5.3 & 8.0 & 2.6 & 2.0 & 1.0 & 2.3 & 0.3 & 0.3 \\
& $0 \%$ & $5 \%$ & $\%$ & $5 \%$ & $6 \%$ & $5 \%$ & $3 \%$ & $8 \%$ & $1 \%$ & $0 \%$ & $4 \%$ & $3 \%$ & $3 \%$ \\
\hline
\end{tabular}

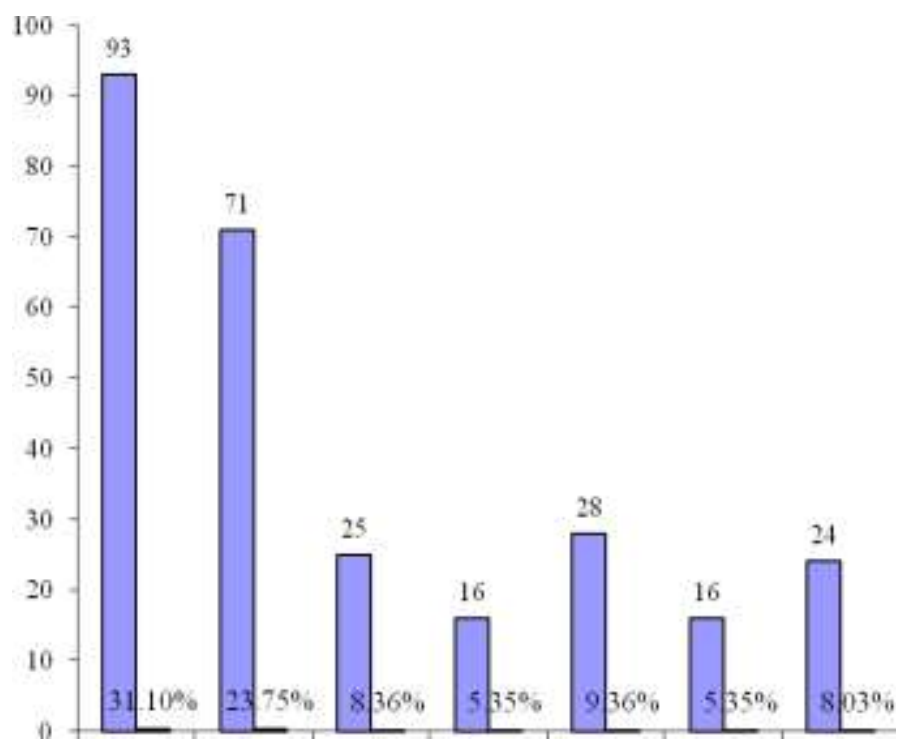

Fig. 1. General distribution of caries of all surveyed children from groups 
Medium and confidence intervals of $\mathrm{dmft}$ and age (Tab. 4).

Table. 4. Medium and confidence intervals of dmft and age

\begin{tabular}{|l|l|l|l|l|l|l|l|}
\hline Descriptive Statistics for Dependent Variable dmft \\
\hline & Level of - Factor & $\mathbf{N}$ & dmft - Mean & dmft - Std.Dev. & dmft - Std.Err & $\mathbf{- 9 5 , 0 0 ~ - ~ C n f . L m t ~}$ & $\mathbf{+ 9 5 , 0 0}$ - Cnf.Lmt \\
\hline Total & & 300 & 3,571906 & 3,709775 & 0,214542 & 3,149697 & 3,994115 \\
\hline Age & 4 & 100 & 3,633663 & 2,855601 & 0,284143 & 3,069932 & 4,197395 \\
\hline Age & 5 & 100 & 1,020408 & 0,824616 & 0,083299 & 0,855083 & 1,185733 \\
\hline Age & 6 & 100 & 6,010000 & 4,480023 & 0,448002 & 5,121066 & 6,898934 \\
\hline
\end{tabular}

In temporary teeth dmft mean 3,57. We found the highest prevalence of cavitated caries in the first and second molars, and the lowest - in the lower second incisors and first incisors, like literature.

There was a statistically significant difference in values for upper and lower jaw - the frequency of dental caries upper jaw is greater than the incidence for mandible $(t=4,1, p<0,05, p=0,0023)$, Fig 2 .

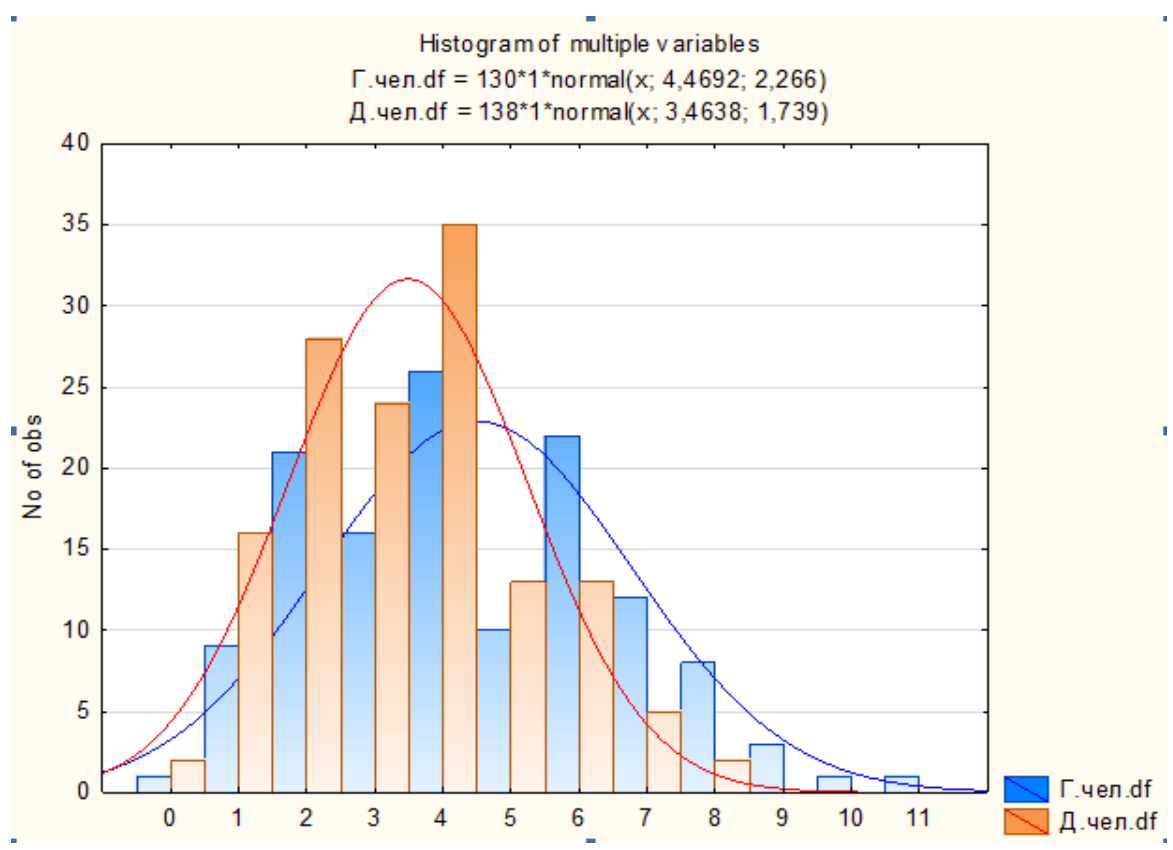

Fig. 2. The Intensity of caries by age in the teeth of the upper and lower jaw-blue line of the upper jaw and red line mandible

Treated deciduous teeth with fillings in this research: Fig. 3.

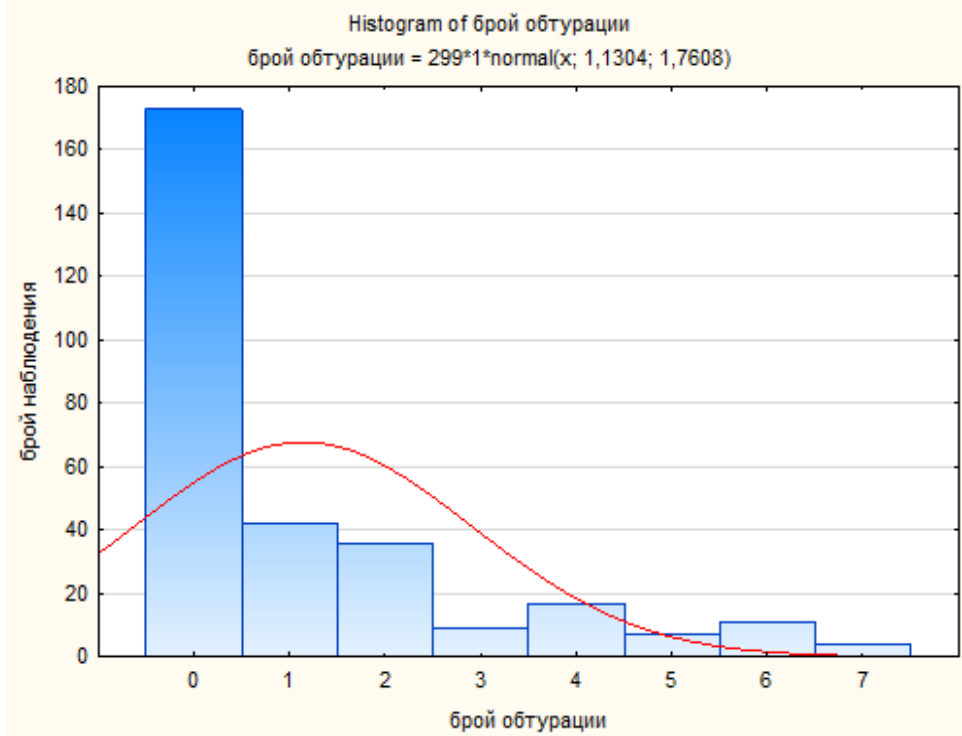

Fig. 3. Frequency distribution of the treated deciduous teeth with fillings 


\section{Discussion}

The study made the following findings: Caries and development of carious lesions is observed from early childhood by children 3 years immediately after the formation of the mixed dentition [4]. The ratio $\mathrm{d} / \mathrm{f}$ is 3d:1f and shows that children are not covered by timely treatment. Most often $\mathrm{d} 1$ and $\mathrm{d} 2$ lesions establish a higher rate on the vestibular surface of the frontal teeth in the throats and the second most affected by vestibular surfaces and necks of temporary canines.

Lower the incidence of dental caries in England [2], Australia, Nepal, Sweden and the USA [1]. Similar Results of our epidemiological investigation are described in North East England, Manchester [2], Costa Rica and Slovakia.

Data $[5,6,7,8]$ confirm the opinion of other authors in Plovdiv [11]. For greater prevalence of caries in deciduous teeth of the upper jaw. Similar results were obtained in scientific studies in other cities of Bulgaria, conducted in previous years $[9,10]$.

\section{Conclusions}

1. Caries is observed from a very early age of 3 years immediately after the formation of the mixed dentition.

2. To the ratio $3 \mathrm{~d} / 1 \mathrm{f}$ we can conclude that children are not covered by timely treatment of early caries lesions.

3. In research done more frequent clinical diagnosis and found $\mathrm{d} 1 \mathrm{a}, \mathrm{d} 1 \mathrm{~b}, \mathrm{~d} 2$ which requires more timely diagnosis and inclusion of children in these groups.

4. Do not diagnose carious lesions of the central and lateral incisors temporary bottom.

\section{References}

[1]. Arruda AL, Sentamaria Kannan R, Inglehart MR et al. Effect of 5\% fluoride varnish application on caries among school children in rural Brazil: A randomized controlled trial. Community Dent Oral Epidemiol. 2012;40:267-275

[2]. Pitts NB, Evans DJ, Nugent Z. The dental caries experience of 5-year-old children in Great Britain: surveys coordinated by the British Society for the Study of Community Dentistry in 1999/2000. Community Dent Health. 2001;18:49-54.

[3]. Prodanova K. Lectures Notices in Statistics. TU-Sofia. 2008; pp. 1-117.

[4]. Holt RD, Joels D, Bulman J, Maddick IH. A third study of caries in preschool-aged

[5]. Children in Camden. Br. Dent. J. 1988;165:67-91.

[6]. Holt RD, Joels D, Winter GB. Caries in preschool children. Br. Dent. J. 1982;153:107-109.

[7]. Kumar JV, Moss ME. Fluorides in Dental Public Healt Programs. Dent Clinam. 2008; 52:387-401.

[8]. Chou R, Cantor A, Zakher B, Mitchell JP, Pappas M. Preventing dental caries in Children, 5 years: systemic review updating USPSTF recommendation. Pediatrics. 2013;132(2):332-35.

[9]. dos Santos AP, Nadanovsky P, de Oliveira BH. A systematic review and meta-analysis

[10]. of the Effects of fluoride toothpastes on the prevention of dental caries in the primary dentition of preschool children. Community Dentistry and Oral Epidemiology. 2013;41 (1):1-12.

[11]. Doichinova L, Peneva M, Bakardjiev P. Assessment of Food Habits in Children 6-12 Years old and the Risk of Caries. Proccedings of 19 Congress of the Bass. 2014 Apr; Serbia, Belgrad;1: 270 p.

[12]. Bozukov H, Ilieva E,Trifonova G, Damyanova D et al. Prevalence of Dental caries Among 5-6 years-old children in Northeastern Bulgaria. Proccedings of 19 Congress of the Bass. 2014 Apr; Serbia, Belgrad;1:278 p.

[13]. Icheva-Goranova A, Kukleva-Todorova M, Rimalovska S. Prevents and Treatment Of non-cavitated carious lesions of primary teeth in children. Proccedings of 19 Congress of the Bass Serbia, Belgrad. 2014 Apr;1(6):276 p. 